\title{
EDITORIAL
}

\section{Evolution of the Paradigms of Mathematics}

The development of mathematics over time has been associated, at different stages, with different phenomena and needs that motivated it, and that were structuring its diverse and continuously evolving nature.

Several thousand years before Christ, humans used mathematics as a tool to quantify the universe that surrounded them. It was, of course, a very rudimentary tool but not for that reason lacking in compelling logic and imagination. For example, it is speculated that primitive humans counted and cared for animals with which they sheltered in caves or caverns, in such a way that when they left the following morning, they knew how many animals were there because they separated a stone for each one of them on their way out, so there were as many animals as there were separated stones. When they returned at sunset, the total number of animals had to match the number of previously separated stones. Perhaps they did not yet understand it, but they were already using the concept of bijective function.

Much later, even before Christ, it was the Geometry of Pythagoras and many others, the primary source of motivation and development of mathematics, which they applied in innumerable different ways to real-life problems.

However, it was in the Modern Age, from the beginning of the XV century to the end of the XVIII century, when a pleiad of great scientists coexisted in the whole range of knowledge accumulated until then, who carried out a major scientific revolution in all possible areas, laying the foundations of Modern Science based on Scientific Knowledge. It was in this scenario where mathematics reached high levels, expanded extraordinarily, both within itself and playing a fundamental role as a powerful modeling tool throughout the entire scientific spectrum. It reached its maximum expressions with Newton, one of the three greatest mathematicians of all time, creator of the infinitesimal calculus, through which he created and built the theoretical bases on understanding the gravitational laws that govern the universe at a macro level.

What type of phenomena, processes, or questions is the mathematical researcher of today trying to understand? What are the new paradigms of mathematics?

The answer is not straightforward because mathematics has spread in almost all possible directions and with countless applications.

Mathematics is the pillar of Science and Technology. Moreover, it is at the center of the development of Man.

However, among the wide range of possibilities, I dare say that one of the great and fascinating challenges of mathematics today is its role as a central tool in prediction processes, both in the past tense and in the future tense. For example, anthropologists, archaeologists when they ask: how and where did our first ancestors originate and live? They try to predict in past time, astronomers and physicists investigate in remote times towards the past (origin of the universe) and also in a future time (final destination of the universe). At the same time, climatologists (e.g., how much rain will fall this year?) and economists (e.g., what will be the value of the dollar in 30 more days?) try to predict behaviors of systems in the future tense, or a virologist wants to know details on the spread of an 
epidemic, such as the one we lived with COVID-19, e.g., when will this pandemic end in Chile? Also, knowing the population dynamics in a particular ecosystem can be a complex problem; for example, is a particular species in a given ecosystem moving towards extinction? In general, scientists have to investigate in one direction or another depending on the question they want to answer.

What does it mean that a particular dynamic is complex? It means that the phenomenon under study is difficult to predict in the long term, and it is possible to predict its state in only a small term (e.g., climatic phenomena).

Mathematically, it means that the function that describes this dynamic is chaotic or presents severe symptoms of being so, for example, sensitivity, which implies that very close points can evolve in discrete time under iteration to very distant places ${ }^{1}$.

Dynamic systems can range from stability to chaos, from simple to very complicated dynamics. When someone wants to study the evolution of a dynamic system, we need to know the equations that govern that system. However, if the system is chaotic, when approximating the different parameters that the equation has, we will have that, precisely because of chaos, small differences in those parameters will make the system evolve differently than it should be.

When we model a system and try to write or determine the equations that govern it, we must make measurements and calculations to find the different coefficients or parameters of the system. However, it is not always possible to find exact solutions, and, in this impossibility, there is a big problem because solutions proximity can lead to a stable or chaotic system. Also, there is an associated problem that is uncertainty, for example, there are times when an exact value is not known, but the range is known.

I would dare to say that great efforts are being made to understand and give better answers to problems that involve the evolution of dynamic systems over time, even in conditions of great difficulty and where there are complex dynamics, chaos, or uncertainty, or even worse all of them.

That would allow us, for example, to predict precisely where a Grade 5 Hurricane will make landfall on its chaotic and unpredictable path to the continent, with devastating effects.

Heriberto Román Flores, PhD. Universidad de Tarapacá

Instituto de Alta Investigación

Arica, Chile

E-mail: hroman@uta.cl

1 H. Román Flores, V. Ayala, C, Chaos Theory, IntechOpenBooks (2018) Chap. 6, pp. 97-114, http//dx.doi.org/10.5772/ intechopen. 72232. Available from: https:/www.intechopen.com/books/chaostheory/chaos-on-set-valued-dynamics-and-control-sets 